\title{
REVISTAACIÓN
}

Revista Educación

ISSN: 0379-7082

ISSN: 2215-2644

revedu@gmail.com

Universidad de Costa Rica

Costa Rica

\section{La argumentación en la formación disciplinar de estudiantes universitarios de Geografía}

Díaz, Juan Pablo; Mignone, Aníbal Marcelo; Roque Bar, Aníbal

La argumentación en la formación disciplinar de estudiantes universitarios de Geografía

Revista Educación, vol. 44, núm. 1, 2020

Universidad de Costa Rica, Costa Rica

Disponible en: http://www.redalyc.org/articulo.oa?id=44060092017

DOI: https://doi.org/10.15517/revedu.v44i1.36687

Esta obra está bajo una Licencia Creative Commons Atribución-NoComercial-SinDerivar 3.0 Internacional 


\title{
La argumentación en la formación disciplinar de estudiantes universitarios de Geografía
}

\author{
Argumentation and Disciplinary Training for University Geography Students
}

Juan Pablo Díaz

Universidad Nacional del Nordeste, Argentina

pablodiaz@hum.unne.edu.ar

(iD http://orcid.org/0000-0003-3822-8927

Aníbal Marcelo Mignone

Universidad Nacional del Nordeste, Argentina

animarmig@hotmail.com

(D) http://orcid.org/0000-0001-9666-7747

Anibal Roque Bar

Universidad Nacional del Nordeste, Argentina

anibalrbar@hum.unne.edu.ar

(iD) http://orcid.org/0000-0002-7381-5425

\author{
DOI: https://doi.org/10.15517/revedu.v44i1 1.36687
http://www.redalyc.org/articulo.oa?id $=44060092017$ \\ Redalyc: http://www.redalyc.org/articulo.oa?id=44060092017
}

Pet//orcid.org/0000-0002-7381-5425

Recepción: 03 Mayo 2019

Aprobación: 16 Octubre 2019

\section{Resumen:}

El presente artículo científico tiene como objetivo describir los modos en que argumenta el estudiantado avanzado de Geografía y, asimismo, comprender cómo los supuestos epistemológicos explícitos y subyacentes en el plan de estudios, orientan la construcción de dichas argumentaciones. El diseño metodológico de la investigación es descriptivo y privilegia el abordaje cualitativo. La muestra estuvo integrada por veintiséis estudiantes de una universidad púbica de la ciudad de Resistencia (Argentina). Las fuentes de recolección de datos fueron el Plan de Estudios de la carrera de Geografía, y el resultado de producciones argumentativas elaboradas por el estudiantado. Se concluye que el plan de estudios, orientado fuertemente hacia la Geografía física, otorga condiciones para la construcción de buenas argumentaciones en dichas temáticas, por parte del alumnado. Se advierte, no obstante, que dichas prescripciones curriculares soslayan otras perspectivas geográficas que habilitarían a una formación más integral de los futuros geógrafos.

Palabras clave: Geografía, Estudiante, Plan de estudios, Universidad.

\section{Abstract:}

The goal of this scientific article is to describe the different types of arguments made by advanced Geography students and understand how explicit and underlying epistemological assumptions of the study plan guide students in constructing these arguments. The methodological design is descriptive with a qualitative scope. The sample included 26 students from the state university of Resistencia, Argentina. Data collection sources included the Geography Major Study Plan and the result of arguments prepared by the students. It can be concluded that the current study plan is well directed at developing proper arguments in this subject matter. It is advised, however, that such curricular requirements do, in fact, undermine other geographic perspectives that are necessary skills for future geographers.

KEYWORDS: Geography, Students, Study Plan, University.

\section{INTRODUCCIÓN}

En los procesos de aprendizaje la argumentación juega un papel fundamental en la construcción de conocimientos. En este sentido, Gómez (2006) plantea que la argumentación es una determinada manera de 
hablar o escribir con el fin de justificar como verdadera una afirmación o enunciado. Por su parte, Cuenca (1995) lo refiere como un fenómeno discursivo que está constituido por una macrofunción, la cual a su vez posee tres funciones básicas: expresa la opinión de quien la emite, intenta influir sobre el receptor de la información, y se manifiesta sobre el mundo.

En el ámbito universitario, trabajar con textos propios del campo disciplinar y científico posibilita al estudiantado el desarrollo de competencias argumentativas. Es el caso de la carrera de Geografía, campo disciplinar donde conviven dos abordajes epistémicos, por un lado, la Geografía física y por otro, la Geografía humana. Puede preverse que dichas perspectivas den condiciones para la construcción de argumentos los cuales expresen matices entre ambas perspectivas geográficas.

Los fenómenos que abordan la Geografía física están determinados causalmente y, por lo tanto, las causas operan como condición suficiente para que el fenómeno ocurra. Así, cabría esperar que las argumentaciones estructuradas con base en la Geografía física, orienten la construcción de tesis y razones en términos claramente determinísticos.

Aquellas argumentaciones generadas sobre contenidos de la Geografía humana, mostrarían mayores márgenes de variabilidad, en tanto los factores condicionantes de sus fenómenos se sustentan fundamentalmente en acciones humanas, definidas en torno de motivaciones e intereses, más que en sucesos físicos. Al decir de Bunge (1997),

... no sería difícil demostrar que la ciencia de la sociedad y de la historia son imposibles, pues en los asuntos humanos no pueden encontrarse dos sucesos idénticos y por otra parte es evidente que en ellos intervienen categorías de determinación no causales, como la estadística, la teleología y la dialéctica (pp. 369-370).

En función de lo expuesto, este trabajo tiene como objetivo describir los modos en que argumenta el estudiantado avanzado de Geografía y asimismo comprender cómo los supuestos epistemológicos explícitos y subyacentes en el plan de estudios, orientan la construcción de dichas argumentaciones.

\section{ANTECEDENTES}

En el campo del conocimiento científico, numerosas investigaciones abordaron la argumentación y el razonamiento en el alumnado como elementos importantes en los procesos de enseñanza y de aprendizaje, al momento de proponer o evaluar estrategias áulicas con el fin de mejorar las competencias argumentativas (Kelly y Takao, 2002; Custodio y Sanmartí, 2005). Por su parte, en el ámbito universitario, existe profusa bibliografía sobre la argumentación en contextos de escritura y alfabetización académica (Arnoux, Di Stefano y Pereira, 2002; De la Barrera y Bono, 2004; Carlino, 2005; De Zerdán; De Sirgo; López, 2010a), siendo mayormente investigada en el campo de la enseñanza de las ciencias exactas y naturales (Bar, 2012; Archila, 2012).

En el campo de las ciencias sociales y humanas (Canals, 2007; Padilla y Carlino, 2010) los estudios sobre la argumentación han tenido menos desarrollo, resultando dificultoso encontrar investigaciones desarrolladas con estudiantes universitarios en proceso de finalización de la carrera, particularmente en el área de la Geografía. Por ello, este trabajo pretende ser un aporte al campo del conocimiento académico, y especialmente, a la disciplina geográfica y a la formación de los futuros profesionales de esta disciplina.

Actualmente la Geografía se ve permeada por una multiplicidad de enfoques que deberían constituirse en ejes de la formación en Geografía. En este sentido, el objetivo de este trabajo es describir los modos en que argumenta el estudiantado avanzado de Geografía, asimismo, comprender cómo los supuestos epistemológicos explícitos y subyacentes en el plan de estudios, orientan la construcción de dichas argumentaciones. La pesquisa se desarrolló en el marco de un proyecto de beca de posgrado denominado La argumentación en contexto formativo. Sus modalidades y sesgos en tres carreras de contenido humanistico. 
La misma corresponde a una Beca Interna Doctoral Cofinanciada. Otorgada por el Concejo Nacional de Investigaciones Científicas y Técnicas en convenio con la Universidad Nacional del Nordeste.

\section{MARCo TEÓRICO}

La argumentación, como otras competencias cognitivas, resulta esencial en el ámbito educativo toda vez que garantiza, no solo la producción y validez del discurso, sino también la puesta en marcha de dispositivos para el desarrollo de ciertas destrezas lógicas.

En este sentido, United Nations Educational, Scientific and Cultural Organization [UNESCO] (1995, 1999) y otros organismos internacionales plantean la importancia de dicha habilidad en los trayectos académicos de los graduados universitarios, a fines de mejorar el dominio disciplinar y la formación para la participación ciudadana. La argumentación constituye uno de los pilares clave en la educación superior y su enseñanza puede ser contemplada en los diversos campos disciplinares, independientemente del objeto de estudio que los ocupa.

La formación supone la adquisición de un mínimo de saberes y prácticas que los futuros egresados deberán poner en juego al momento de la labor profesional, aunque no de modo exclusivo, pues estas competencias serán igualmente útiles en el contexto de su ejercicio de ciudadanía.

Aragón (2007) afirma que la argumentación es la elaboración de un discurso escrito u oral, cuyo propósito es convencer o integrar a otros en una conclusión, una opinión u otras reglas de valores. Asimismo, Camps (1995) sostiene que la actividad argumentativa se encuentra fuertemente ligada con el contexto, los argumentos no se dan en el vacío sino en relación con contenidos específicos, muchos de ellos disciplinares, en el caso de la formación superior. En consecuencia, se trata de una situación comunicativa aplicable a distintos campos del conocimiento (Camps y Dolz, 1995).

En la dinámica del proceso argumentativo, el comunicante busca expresar su punto de vista y justificarlo con la finalidad de convencer al interlocutor (Camps y Dolz, 1995; Van Eemeren, Grootendorst, Jackson y Jacobs, 1997). La enunciación del punto de vista se plasma en la producción de una tesis explícita, o implícita, en el discurso como primer paso del proceso argumentativo.

Kuhn (2010) señala que la argumentación puede ser interpretada como una herramienta orientada a favorecer el desarrollo cognitivo del alumnado al momento de enfrentarse a los textos académicos. Más específicamente, Corcoran, Mosher y Rogat, 2009 (citados en Archila, 2012) conciben esta habilidad como uno de los pilares fundamentales en el proceso de aprendizaje del estudiantado, con el fin de acercarlos a conceptos de carácter científico.

Revel et al. (2005) se abocó a investigar la producción de textos devenidos del uso de analogías entre fenómenos naturales y sociales, tarea que posibilitó al alumnado la construcción de argumentos en el marco de modelos teóricos y sus correspondientes datos empíricos.

Canals (2007), en un estudio desarrollado en escuelas secundarias de Barcelona (España), concluye que el desarrollo de las habilidades argumentativas (textuales y discursivas) en ciencias sociales, promueve el aprendizaje organizado y significativo, y posibilita al estudiantado la construcción de un conocimiento más rico, complejo, relativo y aplicable.

Entre los estudios efectuados en el nivel superior, se puede mencionar el trabajo de Gaviria y Corredor (2011), quienes elaboraron una investigación exploratoria con estudiantes universitarios de pregrado en psicología y terapia física pertenecientes a la Universidad Nacional de Colombia. Los autores desarrollaron un estudio de las habilidades argumentativas a partir de un análisis de textos, identificando buena capacidad para distinguir entre argumentos con evidencias y con explicaciones, asimismo, habilidad para evaluar su pertinencia.

De Zerdán, De Sirgo y López (2010b), en una experiencia ejecutada en el ámbito universitario (Universidad Nacional de Tucumán, Argentina), propusieron al estudiantado la elaboración de ponencias y 
monografías, en diferentes temáticas desarrolladas durante el plan de estudio, con la finalidad de fortalecer sus competencias argumentativas. Los resultados mostraron que efectivamente, se produjeron mejoras en la interpretación de textos leídos, y en la redacción de textos escritos.

Las dimensiones que asume la argumentación dependerán de varios factores, algunos en relación con las competencias del sujeto, y otras en vínculo con características del contenido. Así, Bar (2012) afirma que, en el contexto de la enseñanza, la construcción de argumentaciones se definirá conforme con conceptos y nociones propios del campo disciplinar, constituyentes de su contenido.

En esta línea, Erduran y Jiménez (2007) afirman que las habilidades argumentativas posibilitan al estudiantado cierto desarrollo de criterios epistémicos del campo de alguna disciplina en particular, promoviendo la reflexión sobre el conjunto de actores participantes y el contexto circundante, y desarrollando el pensamiento crítico que habilita el desarrollo de una idea inicial hacia una superior.

En una investigación comparada entre la Universidad Nacional de Tucumán y la Universidad de Buenos Aires, Molina y Padilla (2013) analizaron las producciones argumentativas de estudiantes de las carreras de Letras y Biología pertenecientes a ambas instituciones. Las autoras advierten que el alumnado reconoce y valora las especificidades de los argumentos en una u otra área disciplinar, y consideran que estas juegan un rol importante para aprender los contenidos curriculares de las asignaturas. Dichos resultados dan validez a lo expresado por Toulmin (1979, citado Henao y Stipcich, 2008), quien afirma que el fomento de actitudes críticas en el estudiantado, propicia juzgar, evaluar y decidir objetivamente sobre los contenidos desarrollados en las clases por parte del cuerpo docente.

En el contexto local, Bar (2012) ejecutó una investigación con estudiantes universitarios de la carrera de Biología de la Universidad Nacional del Nordeste, situada en la ciudad de Corrientes, capital de la provincia homónima (Argentina). Las actividades propuestas consistieron en tareas de argumentación y explicación de textos disciplinares. Los resultados mostraron que la formación disciplinar posibilitó al alumnado la elaboración de buenos textos argumentativos y que el buen desarrollo de explicaciones en ese marco solo es posible cuando las teorías disponibles se hallan encuadradas en términos del paradigma vigente en la disciplina.

Si bien la problemática de la argumentación ha sido investigada en diferentes niveles educativos, ha sido escasamente estudiada en el ámbito de la educación superior en Argentina. En tal sentido, y como se mencionó en la introducción, este trabajo pretende constituir un aporte al estudio de las habilidades de la argumentación en Geografía, como disciplina multiparadigmática (Biglan, 1973).

La formación en Geografía ofrece al estudiantado herramientas teórico-conceptuales, metodológicas y prácticas, tanto desde la perspectiva física (hidrografía, geomorfología, etc.) como desde la humana (Geografía económica y política, Geografía de la población, etc.) con el objeto de comprender los fenómenos y las interrelaciones que ocurren en el espacio geográfico, así como poder intervenir para mejorar las condiciones de la población y potenciar el desarrollo de los recursos económicos.

La disciplina geográfica fue evolucionando en las maneras de estudiar el espacio geográfico. Los enfoques imperantes durante la segunda mitad del siglo XX fueron: la Geografía regional; la cuantitativa; la de la percepción y el comportamiento; la humanística; la radical.

La Geografía regional aborda el análisis y la síntesis del espacio atendiendo a las configuraciones naturales que este adopta, y que delimitan áreas diferenciadas entre sí.

La Geografía cuantitativa hace uso de diversas fórmulas matemáticas en la búsqueda de regularidades y modelos de configuración espacial, hecho que actualmente es tangible en el uso de los Sistemas de Información Geográfica.

La Geografía de la percepción y el comportamiento aborda el espacio desde la perspectiva de la subjetividad, es decir, desde las representaciones mentales que los sujetos construyen en torno de su contexto

En la corriente humanista se recupera el concepto de lugar y adquiere prioridad el espacio vivido, el construido desde la experiencia cotidiana e histórica y de las construcciones simbólicas. El enfoque radical 
pone el acento en la transformación del espacio, en tanto producto social sobre el cual el geógrafo deberá adoptar una postura crítica y reflexiva (Capdepón, 2004; Cuadra, 2014).

Si se asume que el plan de estudios, además de lo epistemológicamente explícito, contiene elementos subyacentes, puede afirmarse que su análisis contribuirá a comprender cómo dichos contenidos dan sustento a las argumentaciones construidas por el estudiantado.

\section{Metodología}

El diseño metodológico de la investigación es fundamentalmente descriptivo, pues intenta reconstruir los modos en que argumentan estudiantes de Geografía, a través de categorías propuestas por trabajos de investigaciones anteriores (Cordero, 2000; Bar, 2012). En tanto que las descripciones de las argumentaciones refieren a un campo disciplinar específico como lo es la Geografía, también se pretende dar cuenta de cómo las prescripciones del plan de estudio de esa carrera inciden en esa actividad.

Dichas prescripciones obedecen a motivaciones, intereses y decisiones de los actores responsables de la gestión, como asimismo a las demandas académicas y a las del medio social, todo lo cual requiere ser entendido para encarar las formas argumentativas del alumnado. Esta forma de abordaje inscribe al presente trabajo en una perspectiva de tipo cualitativa, a la vez que comprensiva.

Para esta investigación se obtuvo información de dos fuentes, el Plan de Estudios de la carrera de Geografía, y las argumentaciones elaboradas por veintiséis estudiantes de cuarto y quinto año de la Licenciatura y Profesorado en Geografía de la UNNE. Su selección se sustentó en el supuesto de que dichos sujetos conocían debidamente los contenidos específicos y las operaciones que la disciplina demanda durante la formación, en tanto estos, eran referentes o informantes idóneos para dicho fin. Dicha idoneidad deviene de su formación avanzada en la disciplina lo cual le permitiría disponer de las herramientas conceptuales y metodológicas necesarias para argumentar en el nivel que tendría un egresado reciente.

En cuanto al plan de estudios, se identificaron los subtítulos, y se analizaron los contenidos de la fundamentación, el campo profesional, el perfil del graduado, los alcances del título, los objetivos generales de aprendizaje y objetivos de aprendizaje por ciclos, la distribución de las asignaturas en la estructura curricular adoptada; dado que dichos apartados dan cuenta tanto de cuestiones explícitas como implícitas, en lo que hace al análisis que se propone.

El análisis buscó la reconstrucción de las categorías implícitas o subyacentes operantes en el discurso en el que se expresa el plan. Dichas categorías se estructuraron conforme con las dimensiones epistemológicas y metodológicas del campo disciplinar, algunas expresas, otras tácitas. Por ejemplo, los supuestos que definen objetivos y contenidos de las asignaturas, las funciones que estas cumplen en el currículo; las capacidades que se pretende incorporar en la persona egresada o futuro profesional; entendiendo que estas, entre otras, dan idea de cómo se conforma el objeto geográfico, qué entidad tiene, qué se sabe de él y cómo se sabe, cuáles son sus técnicas de indagación, cómo se construye el trayecto formativo a esos fines, qué se requiere para lograrlo, etc.

Dicho análisis se abordó desde la técnica del análisis de contenido. Particularmente, la técnica semántica y estructural o funcional, cuya meta consiste en desvelar elementos no manifiestos del contenido (significado implícito o latente y connotaciones) (Conte, 2000; Colle, 2011).

A fin de evaluar la calidad de las argumentaciones construidas por el estudiantado, se diseñaron con dos textos descriptivos vinculados con la formación disciplinar del alumnado.

La situación planteada en el texto 1: El desplazamiento de los continentes, consideró al movimiento de los continentes desde la teoría de la Tectónica de Placas, explicada desde la perspectiva física de la Geografía. Por su parte, la situación caracterizada en el texto 2: La agricultura en África, tuvo en cuenta la agrosilvicultura en el Sahel africano, utilizando los aportes del Determinismo (propio de los encuadres de la Geografía Física o Fisiogeografía) y el Posibilismo geográfico (afín a las Geografías Humanas o Antropogeografía), ambas 
ramas de la Geografía (Cuadra, 2013). Sendas perspectivas incluyen a la mayoría de las subdisciplinas sobre las cuales se organizan los diseños curriculares para las carreras de Geografía. La Fisiogeografía involucra a: Meteorología, Química, Hidrología, entre otras; en tanto que, la Antropogeografía comprende: Economía, Antropología, Sociología, entre otras (Ordóñez, 1991).

La consigna para el análisis de dichos textos solicitó las explicaciones de las situaciones descriptas y la identificación de las tesis y las razones de las argumentaciones expuestas en dichos textos. Se requirió, también, valorar los argumentos esgrimidos en algunas de las tres categorías siguientes: débil, moderada o sólida. La primera refiere a evidencias controvertibles o debatibles que no alcanzan para una justificación plena. La segunda categoría, se vincula con razones aceptadas disciplinarmente, pero sin consenso unánime, debido a criterios ya superados, o bien, novedosos, pero sin suficiente debate.

La última, se relaciona con formas validadas, y que constituyen parte de lo que Cuadra (2013) las llama ramas de la Geografía actual: Geografía Física o Fisiogeografía y Geografías Humanas o Antropogeografía.

Para clasificar las producciones de estudiantes, se adecuaron las categorías propuestas en el estudio de Bar (2012), cuyo propósito fue examinar los niveles de argumentación en estudiantes avanzados de la carrera de Biología, las cuales son:

Lo referido a las tesis: Explicaciones por referencia a leyes o teorías actuales; Explicaciones referidas a conceptos muy generales o de aplicación difusa; Explicaciones que abrevian en concepciones anacrónicas o del sentido común; Explicaciones que refieren a simplificaciones de leyes o teorías vigentes; Explicaciones basadas en conceptos erróneos o mal aplicados.

Lo concerniente a las razones: Razones sustentadas en la evidencia empírica; Razones que atacan la validez de las razones (son nuevas razones, que niegan las razones propuestas en el texto); Pseudorrazones inspiradas en agentes causales; Pseudorrazones que reproducen información textual.

Lo concerniente a las razones: Razones sustentadas en la evidencia empírica; Razones que atacan la validez de las razones (son nuevas razones, que niegan las razones propuestas en el texto); Pseudorrazones inspiradas en agentes causales; Pseudorrazones que reproducen información textual.

En caso de identificar en el discurso, elementos no concordantes con los descriptos por Bar (2012), se propondrán nuevas categorías, o se restructurarán algunas de ésas.

Posteriormente, las argumentaciones generadas por el alumnado se clasificaron según la metodología de Cordero (2000), en las siguientes categorías: texto sin tesis, 1 punto (nivel nulo); tesis implícita o explícita sin relación con el tópico, 2 puntos (nivel bajo); tesis implícita en relación con el tópico, 3 puntos (nivel regular o satisfactorio); y reconocimiento de la tesis explícita en relación con el tópico, 4 puntos (nivel óptimo).

\section{Resultados}

En este apartado se presentarán los resultados obtenidos de ambas fuentes de datos: A) el plan de estudios; B) las producciones de estudiantes en relación con los dos textos disciplinares; en primera instancia con las categorías de Bar (2012), y posteriormente, de Cordero (2000).

\section{A) El Plan de estudios de la carrera}

El plan de estudio de Geografía fue aprobado en el año 2000, y a la fecha no tuvo ninguna modificación de su estructura. El análisis estará centrado en el contenido de algunos de los apartados que constituyen dicho plan.

En la Fundamentación se plantea que la Geografía tiene como objetivo el análisis del espacio, y que esta fue evolucionando en la forma de abordar el espacio en función del desarrollo tecnológico y socioeconómico de las sociedades. Aquí se hace mención al crecimiento acelerado de la población mundial, el "agotamiento de los recursos naturales, nuevos problemas del medio-ambiente y ecológicos [ante lo cual la Geografía debe 
seguir] jugando el protagonismo que le corresponde ante este nuevo desafío" (Facultad de HumanidadesUniversidad Nacional del Nordeste [UNNE], 2000, p. 19).

Es importante rescatar la historia de la carrera, es decir la institucionalización de la Geografía en la UNNE. En primera instancia esta se organiza como Departamento el que se constituye con cada una de las cátedras, que dictan las diferentes materias del plan. En años sucesivos, la función de investigación toma cuerpo y se fortalece, objetivándose en el trabajo de docentes investigadores, cuyos hallazgos son publicados en congresos, en libros y revistas científicas locales, nacionales o internacionales. La materialización de este nuevo rol académico se da en el marco de los institutos, los cuales se hallan normados en los aspectos que hacen a su gestión, su organización, sus misiones y sus funciones.

En el Campo profesional se expone el objeto del trabajo del geógrafo, que comprende la docencia en el nivel universitario y la ejecución de tareas de investigación. Además, señala la importancia de las actividades de extensión y asesoramiento a terceros. Por ello, el futuro egresado debe: "Realizar tareas de consultoría, relevamientos y supervisiones técnicas para organismos gubernamentales y no gubernamentales, empresas estatales y privadas e Intervenir en el ordenamiento territorial, ya que esta actividad cobraba mayor auge en los países latinoamericanos y en Argentina” (Facultad de Humanidades-UNNE, 2000, p. 20).

En el Perfil del graduado se explicitan distintas capacidades, actitudes, habilidades, aptitudes que deben tener los egresados, como por ejemplo: “...un sólido conocimiento de la disciplina y actitud científica respecto a los hechos geográficos, mantener la ética profesional y el compromiso social, trabajar en forma flexible e interdisciplinariamente con otros campos para generar conocimientos" (Facultad de Humanidades-UNNE, 2000, p. 20).

Menciona la capacidad para "la elaboración de cartografías con base a la aplicación SIG e interpretación de fotografías áreas, imágenes satelitales y resultados del SIG” (Facultad de Humanidades-UNNE, 2000, p. 20).

Es importante resaltar que no se hace alusión explícita a la habilidad y actitud crítica, así como a la postura reflexiva, elementos requeridos en la investigación y en las participaciones sociales donde intervenga el futuro profesional.

En Alcances del titulo se exponen las actividades que pueden desarrollar los egresados de la carrera. En este apartado se observa una variedad de tareas que puede efectuar el egresado en Geografía: docencia e investigación en nivel superior, capacitación y asesoramiento a terceros, asesoramiento pedagógico y técnico a organismos tanto público como privado.

Los Objetivos generales de aprendizaje están centrados en la adquisición de saberes disciplinares y en la formación de profesionales para la participación en docencia e investigación. La carrera apunta a fortalecer la investigación en busca de la excelencia en la formación del egresado con un amplio y profundo conocimiento de los cuerpos teóricos, con capacidad para transmitir los conocimientos geográficos de forma práctica, amena y comprensible a los individuos y la sociedad.

Los Objetivos de aprendizaje según estructura curricular adoptada (ciclos): primer ciclo instrumental (1er año) propenden a los conocimientos de conceptos y de procedimientos. Los conceptos refieren al objeto de estudio de la ciencia, el espacio geográfico y sus caracteres, las corrientes del pensamiento de la Geografía, el Sistema Solar y fenómeno terrestres, origen, evolución y explicación del relieve terrestre, entre otras. Los procedimientos se vinculan con el manejo de cartografía y escalas, matemática y cálculos estadísticos, búsqueda de fuentes en web y diferentes formatos digitales, entre otras.

En el segundo ciclo de formación profesional básica (2do a 4to nivel) se propone que cada estudiante reconozca e interprete las interrelaciones entre ser humano/medio, sociedad/ambiente, sus respuestas y resultados históricos y actuales sobre la superficie terrestre en diversas escalas geografías.

En el tercer ciclo formación profesional en investigación (5to nivel) continúan con el desarrollo e integración de los conocimientos físicos y humanos en los diferentes seminarios, vinculación la cual permite la formación del futuro investigador en este campo específico. Las dos materias optativas tienen que tener 
vínculo con el tema de la tesis de grado, lo que a veces significa que el profesorado tiene que cursar la/s materia/ $s$ en otras facultades de la UNNE.

En lo que se refiere a la Distribución del total de la asignatura según la estructura curricular adoptada, se explicitan los siguientes ciclos y sus contenidos, según se puede apreciar en la Tabla 1:

TABLA 1

Distribución del total de las asignaturas según la estructura curricular de la carrera de Geografía

\begin{tabular}{|c|c|c|}
\hline Ciclos & Asignaturas & Algunos contenidos \\
\hline Primer ciclo & $\begin{array}{l}\text { Introducción a la Geografia } \\
\text { (desarrollo epistemológico); } \\
\text { Técnicas en Geografia I; } \\
\text { Geomorfologia; Normativa } \\
\text { Castellana; Matemática Aplicada; } \\
\text { Introducción a la computación. }\end{array}$ & $\begin{array}{l}\text { "El espacio geográfico y su } \\
\text { carácter; Tratamiento y } \\
\text { cartografiado de los datos } \\
\text { geográficos (SurFer); } \\
\text { Relación entre la Geología y } \\
\text { la Geomorfología, entre } \\
\text { otras" (Facultad de } \\
\text { Humanidades-UNNE, 2000, } \\
\text { pp. 23-25). }\end{array}$ \\
\hline Segundo Ciclo & $\begin{array}{l}\text { Climatologia; Geomorfologia Il; } \\
\text { Hidrografia Marina y Continental; } \\
\text { Biografia y Geografia Ambiental; } \\
\text { Sensores Remotos; Geografia de la } \\
\text { Población; Geografia Económica y } \\
\text { Politica General; Geografia Urbana } \\
\text { y Agraria; Geografia y Sociedad; } \\
\text { Técnicas en Geografia II; Teoria y } \\
\text { Métodos de investigación; Geografia } \\
\text { Argentina; Geografia de América; } \\
\text { Geografía de Europa y Asia; } \\
\text { Geografia Africa y Oceania; } \\
\text { Seminario de Investigación del } \\
\text { Nordeste Argentino. }\end{array}$ & $\begin{array}{l}\text { Estudio geográfico de los } \\
\text { climas; Relieve litoral y } \\
\text { submarino; Movimiento de } \\
\text { los océanos; Medidas } \\
\text { hidrométricas y } \\
\text { terminología básica; } \\
\text { Sistemas fotográficos de } \\
\text { recolección de datos; } \\
\text { Geografía de la población; } \\
\text { Geografía económica de la } \\
\text { producción; La ciudad como } \\
\text { fenómeno espacial; Modos } \\
\text { de segregación y su } \\
\text { recuperación en la } \\
\text { estructura territorial; La } \\
\text { carta temática como sistema } \\
\text { de comunicación; La } \\
\text { estructura regional del } \\
\text { territorio; entre otros } \\
\text { (Facultad de Humanidades- } \\
\text { UNNE, 2000, pp. 25-32). }\end{array}$ \\
\hline Tercer Ciclo & $\begin{array}{l}\text { Seminario de Fisiografía; Seminario } \\
\text { de Geografía Humana; Seminario de } \\
\text { Geografía; Materias optativas y } \\
\text { Tesis de Licenciatura }\end{array}$ & $\begin{array}{l}\text { Algunos de los contenidos } \\
\text { enunciados son: "El croquis } \\
\text { de Geografía Física: } \\
\text { Elaboración y explicación; } \\
\text { La Geografía regional, } \\
\text { objetivos y características } \\
\text { conceptuales, entre otras" } \\
\text { (Facultad de Humanidades- } \\
\text { UNNE, 2000, pp. } 32-34 \text { ). }\end{array}$ \\
\hline
\end{tabular}

Fuente: Elaboración propia

\section{B. Análisis de las producciones del estudiantado (Geografía física y Geografía humana)}

Del total de categorías utilizadas por Bar (2012), en este trabajo solo se presentaron las que se consignan en la Tabla 2: 
TABLA 2

Categorías de tesis y razones, según la clasificación de Bar (2012), identificadas en las argumentaciones construidas por las y los estudiantes, en relación con el texto 1

\begin{tabular}{lll}
\hline Texto 1 & & \\
\hline & Tesis & Razones \\
\cline { 2 - 3 } & $\begin{array}{l}\text { Tesis referidas a leyes o teorias } \\
\text { actuales. }\end{array}$ & $\begin{array}{l}\text { Razones sustentadas en la } \\
\text { evidencia empirica. }\end{array}$ \\
\cline { 2 - 3 } Tesis referidas a conceptos muy & $\begin{array}{l}\text { Pseudorrazones que } \\
\text { reproducen la información } \\
\text { textual. }\end{array}$ \\
\cline { 2 - 3 } & $\begin{array}{l}\text { generales o de aplicación difusas. } \\
\text { Tesis basadas en conceptos erróneos }\end{array}$ & $\begin{array}{l}\text { Pseudorrazones inspiradas en } \\
\text { agentes causales. }\end{array}$ \\
\cline { 2 - 3 } & o mal aplicados. & \\
\cline { 2 - 3 } & $\begin{array}{l}\text { Pseudotesis que reproduce } \\
\text { información textual. }\end{array}$ & \\
\hline
\end{tabular}

Fuente: Elaboración propia

De las categorías generada por Bar (2012), solo pudieron identificarse aquellas que se detallan en la Tabla 3.

TABLA 3

Categorías de tesis y razones, según la clasificación de Bar (2012), identificadas en las argumentaciones construidas por las y los estudiantes, en relación con el texto 2

\begin{tabular}{|l|l|l|}
\hline Texto 2 & Tesis & Razones \\
\cline { 2 - 3 } & $\begin{array}{l}\text { Tesis referidas a leyes o teorias } \\
\text { actuales. }\end{array}$ & $\begin{array}{l}\text { Razones sustentadas en la } \\
\text { evidencia empirica. }\end{array}$ \\
\cline { 2 - 3 } Categorías & $\begin{array}{l}\text { Tesis referidas a conceptos muy } \\
\text { generales o de aplicación difusas. }\end{array}$ & \\
\cline { 2 - 3 } & $\begin{array}{l}\text { Tesis que refieren a simplificación de } \\
\text { leyes o teorias vigentes. }\end{array}$ & \\
\cline { 2 - 3 } & $\begin{array}{l}\text { Pseudotesis que reproducen } \\
\text { información textual. }\end{array}$ & \\
\hline
\end{tabular}

Fuente: Elaboración propia

Texto 1 : El desplazamiento de los continentes. Identificación y valoración de tesis y razones a partir de las producciones elaboradas por las y los estudiantes.

La mayoría de los participantes identifica Tesis referidas a leyes o teorías actuales, es decir, reconoce a la misma en términos de la separación y el desplazamiento de los continentes como resultado del movimiento de las placas tectónicas, visible en la superficie terrestre mediante diferentes evidencias físicas. Algunos ejemplos al respecto son:

La formación de los actuales continentes y el relieve continental son producto del sistema de corriente del manto, como es el caso de la aparición del océano Atlántico por una corriente ascendente en el medio del Pangea, como también la formación de la gran dorsal del centro del Atlántico (S. L. Meier, comunicación personal, 11 de abril, 2018).

La creación de las dorsales oceánicas debido al ascenso del material proveniente del interior de la corteza terrestre, la presencia de islas volcánicas sobre el borde de contacto entre las placas (S. T. Martínez, comunicación personal, 10 de mayo, 2018).

Durante la formación comprendí que muchos de los fenómenos físicos que se dan en superficie se sustentan en procesos geológicos. La deriva continental se basa en la teoría de tectónica de placas que dice: el planeta está dividida en placas, estas convergen o divergen. La convergencia de placas provoca que el material en superficie o en el lecho oceánico se eleve, se pliegue (O. R. Sánchez, comunicación personal, 22 de junio, 2018). 
En el primer y segundo caso, el alumnado establece que los movimientos de las placas tectónicas que se encuentran en el interior del planeta, han generado movimientos convectivos (corrientes del manto) los cuales provocaron la formación de las dorsales submarinas en el fondo del océano Atlántico, con aparición de nuevas tierras que produjeron la separación de los continentes y el crecimiento de dicho océano. Cabe aclarar que este proceso continúa en la actualidad.

En el tercer caso, se puede apreciar que el estudiantado reconoce la teoría de la Tectónica de Placas, como el mecanismo que provoca el desplazamiento de los continentes; esta teoría aparece implícita en el texto y de acuerdo con las razones expuestas el estudiantado recuperó esos datos aprendidos y alcanzó un análisis adecuado, en virtud a su nivel de formación académica.

En relación con la identificación de las tesis, una minoría de los entrevistados generó discursos en los cuales se han detectado básicamente tres tipos de dificultades. Por un lado, la Tesis referidas a conceptos muy generales o de aplicación difusas, como puede notarse en las siguientes producciones:

El desplazamiento de los continentes se produce por la conjunción de varios fenómenos y factores, que son: el empuje del océano Atlántico y por otro lado, el océano Pacífico con el corrimiento de manto que circula hacia América (A. M. Ortiz, comunicación personal, 25 de abril, 2018).

La teoría de tectónica de placas es la que explica el movimiento de los continentes (P. Cohen, comunicación personal, 12 de marzo, 2018).

En el primer caso, el alumnado considera que el desplazamiento de los continentes se debe al empuje de los océanos Atlántico y Pacífico y a las corrientes del manto que llegan al continente americano. Como se mencionó anteriormente, son los movimientos convectivos los que contribuyen al desplazamiento de los continentes y al crecimiento o estrechamiento de los océanos. En la segunda situación, el alumnado reconoce y enuncia la teoría de la Tectónica de Placas como generador del desplazamiento, pero no explica la misma.

Se advierten también, Tesis basadas en conceptos erróneos o mal aplicados. Un ejemplo de ello es:

Para mí las placas se fragmentaron y a medida que pasaban los años se fueron separando (M. T. Pérez, comunicación personal, 23 de marzo, 2018).

Puede apreciarse que los términos utilizados por este estudiante no son adecuados en términos de la teoría invocada, pues las placas no sufren procesos de fragmentación, y son los continentes los que la experimentan. Además, la explicación remite a períodos expresados en años, lo que es erróneo dado que los fenómenos aludidos se generan en el trascurso de millones de años (eras y períodos).

Por último, se observa Pseudotesis que reproduce información textual se trata de una nueva categoría no identificada en el trabajo de Bar (2012). Un ejemplo es:

En el manto, por debajo de la corteza terrestre, existen corrientes de convergencia y divergencia que configuran el relieve en la superficie (J. M. Gómez, comunicación personal, 28 de mayo, 2018).

En este caso, se puede advertir que el estudiantado solo copió literalmente una parte del texto, sin reconocer la tesis asociada con la tectónica de placas y la deriva continental; por lo tanto no se trata de una verdadera argumentación.

En cuanto a la identificación de las razones, la mayoría de los entrevistados reconocen las Razones sustentadas en la evidencia empirica, dadas en hechos físicos que pueden observarse en la superficie terrestre; un ejemplo de esto es:

Las innumerables pruebas científicas realizadas por geólogos, vulcanólogos, sismólogos y otros científicos del área que explican que geodinámica interna de la tierra demuestran que este proceso es factible. Los ejemplos están a la vista. El valle del Riff en África es el mejor ejemplo de este proceso de separación continental y como se produce, evocando vulcanismo y sismos. (S. T. Martínez, comunicación personal, 10 de mayo, 2018).

Una minoría de estudiantes no reconoce las razones. Algunos de ellos plantean Pseudorrazones que reproduce la información textual. Un ejemplo de ello: 
La aparición del océano atlántico supone que una corriente ascendente en el medio del Pangea ha empujado a una parte y a otra los continentes americanos y europeos (R. T. Pérez Ruiz, comunicación personal, 25 de junio, 2018).

Otro elemento detectado son las Pseudorrazones inspiradas en agentes causales, como puede notarse en las siguientes formulaciones:

Las razones que sustentan la tesis o afirmaciones son los diferentes cambios y movimientos que se produjeron en la superficie terrestre, los cuales fueron adquiriendo la forma que tiene actualmente (A. Daniel, comunicación personal, 20 de marzo, 2018).

La razón principal que la sustenta es la existencia de la constante acción de los procesos internos de la corteza terrestre, puesto que la energía calórica que proviene del interior del planeta se renueva constantemente (se compensa) y los movimientos siguen su ciclo (M. F. Sorin, comunicación personal, 28 de junio, 2018).

Por último, la mayoría de estudiantes expresa una valoración moderada sobre las razones expuestas.

Texto 2: La agricultura en África. Identificación y valoración de tesis y razones a partir de las producciones elaboradas por las y los estudiantes.

Como ocurriera en el tratamiento del primer texto, la mayoría de los participantes identifican las Tesis referidas a leyes o teorias actuales. De esta manera, reconoce la influencia climática y morfológica de la zona referidas en el texto (determinismo geográfico), y a la posibilidad de desarrollar la agrosilvicultura en un área con limitaciones físicas (posibilismo geográfico). Ejemplos de ellos son:

El Sahel está ubicado en el sur del desierto de Sahara en una transición de clima desértico y ecuatorial, por lo cual esta región presenta períodos extraordinarios donde se producen abundantemente precipitaciones y períodos de escasas lluvias por lo cual, los agricultores de la región se adaptan a las inclemencias del clima, mediante el desarrollo de la agrosilvicultura (M. T. Pérez, comunicación personal, 23 de marzo, 2018).

El desarrollo de la agrosilvicultura aumentó la producción agrícola a pesar de las desfavorables condiciones ambientales. Esta situación se debe al uso de sistemas agrícolas resistentes a la sequía que permite una mayor producción. A dichos mecanismos se acude debido a que las condiciones climáticas y edafológicas no permiten un desarrollo adecuado de los cultivos (K. Bordon, comunicación personal, 7 de mayo, 2018).

La agricultura en África resultó dificultosa por ser un continente con gran extensión de suelo árido. Haciendo referencia al territorio del Sahel, los agricultores del lugar deben adaptarse a las situaciones hostiles de la naturaleza ya que hay períodos de extraordinarias lluvias y otras de grandes sequías. Una de las opciones, para mejorar las condiciones económicas de la población del lugar, es la implementación de la agrosilvicultura, la cual es de bajo costo (D. A. Ruggieri, comunicación personal, 19 de abril, 2018).

En las tres producciones se identifican claramente todos los elementos que hacen parte de la tesis.

En relación con la identificación de las tesis, una minoría de estudiantes evidencian dos dificultades en sus producciones, a saber: Tesis referidas a conceptos muy generales o de aplicación difusas como se expresan en el siguiente ejemplo:

La revolución verde ha tenido un impacto a escala mundial posibilitando la expansión de la frontera agrícola en todo el mundo (M. L. Torres, comunicación personal, 14 de marzo, 2018).

El alumnado refiere al impacto del fenómeno de la revolución verde en el continente africano sin atender al planteo específico de la agrosilvicultura, como se desarrolla en el texto.

Una segunda dificultad, se expresa en las Tesis que refieren a simplificación de leyes o teorías vigentes, tal el caso de:

África es un continente sumamente árido y el Sahel, una parte del año se ve afectado por lluvias que alivian a los productores con escasas inversiones, que además muchas veces tienen que pagar impuestos por trabajar las tierras donde no son propietarios (Q. Cuadrelli, comunicación personal, 5 de abril, 2018).

Se observa un proceso simplificado donde los períodos de lluvias en el Sahel alivian la situación de las comunidades dedicadas a las actividades agrícolas, muchas de ellas en condiciones de carencias críticas; pero el argumento no condice con el paradigma posibilista planteado en el instrumento. 
El ejemplo que se presenta a continuación muestra que el estudiantado realizó una copia textual del instrumento, por lo tanto, no elaboró la tesis que sustenta su argumentación, lo que se encuadra en la categoría de Pseudotesis que reproduce información textual. Se trata de una nueva categoría no identificada en el trabajo de Bar (2012). Un ejemplo es:

África es uno de los continentes con mayor extensión del suelo árido del planeta, al mismo tiempo que la actividad agrícola es una de los pilares de la economía (S. K Mieres, comunicación personal, 8 de marzo, 2018).

En lo concernientes a la identificación de las razones, puede apreciarse que la mayoría de los entrevistados menciona las evidencias que sustentan la tesis, un ejemplo de esto es:

El determinismo geográfico, en el Sahel, condiciona la actividad económica del hombre en donde la pobreza del suelo y las escasas lluvias condicionan la agricultura.

Existen ecosistemas resistentes a la sequias, en donde se desarrollan una actividad económica que años atrás no se realizaba, se puede decir que el hombre puede modificar su entorno (K. Bordon, comunicación personal, 7 de mayo, 2018).

También aparecen Razones sustentadas en factores incidentes no citados en el texto, se trata de una nueva categoría no identificada en el trabajo de Bar (2012). Ejemplos de esto son:

Las razones son que el imperialismo avanzó sobre ellos y luego el capitalismo, por lo tanto jamás le permitieron desarrollarse (África). Además, debido a sus necesidades y a sus políticos que son bastante corruptos. (O. R. Sánchez, comunicaciones personales, 22 de junio, 2018).

En la evolución histórica del continente, se establecen que las cuestiones sociopolíticas y el accionar de un sistema económico, han jugado un papel preponderante en las condiciones socioeconómicas actuales de los estados africanos.

Razones: ubicación cerca de los ríos. Sistema de riego. Paquetes tecnológicos. Intervención de empresas multinacionales (D.

A. Cordia, comunicación personal, 18 de junio, 2018).

Los conceptos mencionados en este ejemplo aluden una serie de factores y procesos que han favorecido las actividades agrícolas en todo el continente africano, evidencias que no son planteadas en la propuesta de trabajo.

Por su parte, una minoría de estudiantes no reconoce las razones, evidenciando dificultades al momento de identificar evidencias que apoyen su tesis. En tal sentido, se reconocen Razones referidas a simplificaciones de leyes o teorías vigentes, siendo esta una categoría emergente no incluida en la propuesta por Bar (2012). Ejemplo de esta es:

Las razones que se pueden considerar, son las siguientes: una fuerte influencia política, una demanda internacional por alimento, una economía de mercado que obliga a estos países a implementar estos tipos de sistemas, el aumento de la población, de la pobreza, del hambre y debido a un factor climático que permite en cierta época del año, realizar este tipo de cultivo (S. S. Lauría, comunicación personal, 14 de junio, 2018).

En la respuesta brindada, se observa la mención de factores políticos y demográficos que han determinado las características actuales del continente africano, pero no indica las estrategias aplicadas para el desarrollo de la agricultura.

Otro ejemplo para la misma categoría es:

Pese a las malas condiciones que presenta el continente africano, se pudo desarrollar técnicas que optimicen su producción (S. L. Meier, comunicación personal, 11 de abril, 2018).

En líneas generales, la valoración que efectúa el alumnado respecto de la relevancia de las razones identificadas es moderada, al igual que en el caso del primer texto trabajado.

Siguiendo con el análisis de las producciones, tomando la metodología de Cordero (2000), la siguiente tabla muestra las categorías de niveles de identificación de las tesis: 
En la Tabla 4, muestra la distribución de frecuencia de las producciones escritas del estudiantado según el grado de explicitación de las tesis propuestas.

TABLA 4

Niveles de calidad de las producciones escritas por las y los estudiantes de Geografía

\begin{tabular}{lllll}
\hline $\begin{array}{c}\text { Nivel de } \\
\text { explicación de la } \\
\text { tesis }\end{array}$ & $\begin{array}{c}\text { Texto 1: } \\
\text { El desplazamiento } \\
\text { de los continentes }\end{array}$ & $\begin{array}{l}\text { Texto 2: } \\
\text { La agricultura en Africa }\end{array}$ & \\
\cline { 2 - 5 } & $\mathrm{n}$ & $\%$ & $\mathrm{n}$ & $\%$ \\
\hline Nivel nulo & 0 & 0 & 3 & 11 \\
\hline Nivel bajo & 4 & 15 & 3 & 11 \\
\hline Nivel satisfactorio & 7 & 27 & 11 & 43 \\
\hline Nivel óptimo & 15 & 58 & 9 & 35 \\
\hline N & 26 & 100 & 26 & 100 \\
\hline
\end{tabular}

Fuente: Elaboración propia

Se advierte mejores producciones en relación con los contenidos de Geografía física, lo cual daría cuenta de la incidencia que tienen esos conocimientos en el plan y el tratamiento de los mismos en la formación. Lo expresado se manifiesta particularmente en los resultados obtenidos, en los niveles Nulo y Óptimo de dichas producciones. Con respecto a las competencias críticas y reflexivas que debería tener el estudiantado para la elaboración de argumentaciones; de la lectura del plan de estudios no puede inferirse que estas hayan sido trabajadas suficientemente, por cuanto no se hallan mencionadas en ninguno de los apartados analizados del Plan de Estudio (Facultad de Humanidades-UNNE, 2000).

\section{CONCLUSIONES}

Se advierte que las construcciones de argumentos por parte del estudiantado de Geografía; no obstante, algunos errores observados, son en general de buena calidad, lo que se desprende de los análisis efectuados en términos de las clasificaciones de Cordero (2000) y Bar (2012). Dicha calidad viene dada por la correcta identificación de tesis y razones, y la construcción de explicaciones sobre esos contenidos. Dicho resultado es coincidente con lo hallado por Bar (2012) en estudiantes de la carrera de biología.

Puede suponerse que, de modo similar a este, la calidad argumental viene dada por el desarrollo del plan de estudios que otorga condiciones para que las teorías disciplinares desarrolladas durante los procesos formativos sean suficientes para la elaboración de argumentos consistentes (Cordero, 2000; Larraín, Freire, Moretti, Requena, Sabat, 2015).

Puede concluirse que los argumentos construidos por estudiantes de Geografía se hallan condicionados por dos factores dados en la formación; por un lado, los contenidos disciplinares en relación con enfoques y perspectivas fundadas en las materias de la carrera, y por otro, las competencias cognitivas sustentadas en los procesos instruccionales. En este sentido, el análisis del plan de estudios provee información que ayuda encuadrar la formación general del alumnado (Castro y Sánchez, 2013).

Lo que el análisis del plan muestra es que, a pesar de la identificación de problemas emergentes reseñados en su fundamentación, este se encuadra en una perspectiva que no logra desanclarse de la vieja tradición regionalista que aborda el espacio, acudiendo por una parte a su estructura física, y por otra, a los análisis propiamente demográficos de la llamada Geografía humana.

Las materias del segundo nivel operan como propedéuticas para entender cómo los procesos geológicos, hidrográficos, climáticos, entre otros, inciden y determinan el relieve, dando condiciones a las distintas formas que asume el espacio físico. 
De modo similar a las asignaturas del segundo nivel, las del tercero se definen en torno de contenidos donde lo entendido como humano transita por un único camino, definido este por un marco fundamentalmente político y económico, donde lo singular y subjetivo no tiene cabida a la hora de interpretar los vínculos que el hombre establece con el territorio.

Se pretende que lo dado en los dos niveles anteriores, sirva para que en el cuarto esos contenidos se integren en el abordaje de las geografías de los distintos continentes. Así, el tratamiento de cada región se entenderá como una Geografía física que condiciona el quehacer humano, y al hombre como quien responde a dichos condicionamientos, acudiendo a estrategias y artefactos enmarcados en sucesos sociopolíticos y económicos. Dicha perspectiva soslaya los aspectos simbólicos puestos en las interacciones sociales; los discursos de los actores; y los sentidos que los sujetos otorgan al espacio físico. Esta mirada concibe al territorio como mero soporte de las acciones humanas, sin tener en cuenta otras dimensiones del tipo sociales o culturales (Capdepón, 2004; Cuadra, 2014).

Cuando en su fundamentación, se hace referencia a los problemas medioambientales, se espera que el plan de estudios incorpore la temática de modo pleno en el abordaje de cada uno de los territorios estudiados, sin embargo, solo la asignatura Biogeografía y Geografía ambiental la trata, lo que limita dichos contenidos a una única materia, en vez de asumirlos como transversales.

Así, puede inferirse que la capacidad argumentativa del estudiantado de Geografía se incrementa conforme con la clase de contenidos involucrados, de mayor calidad cuando la tesis focaliza en conceptos propios de la Geografía física, de menor, cuando esta aborda temáticas más sociales; y que esta situación se define en torno de las particularidades del currículo desarrollado en la formación (Backhoff, Velasco, y Peón, 2013).

Si bien el plan de estudios no hace explícitas las competencias cognitivas que se promueven, en el perfil del graduado se alude a la asunción de objetividad y de actitud científica, al compromiso social, entre otras, habilidades que suponen el ejercicio de la crítica y la reflexividad con lo cual se asume que el estudiantado transitará su proceso formativo orientado a esos fines. Algo que llama la atención es que, entre las capacidades nominadas, se hace referencia a la interpretación cartográfica en el marco de los sistemas de información geográfica (SIG), actividad meramente instrumental que no guarda relación con competencias cognitivas de orden superior.

Si el pensamiento crítico y reflexivo está presente en los procesos formativos, este parece condicionado por el contenido, toda vez que las argumentaciones se expresan menos elocuentes cuando las nociones en análisis se encuadran en temáticas vinculadas con los aspectos sociales del territorio.

Como corolario puede afirmarse, que no obstante los nuevos requerimientos demandados a la carrera, y que se hallan explícitos en su fundamentación, la formación mantiene cierta distancia de abordajes más complejos, aquellos que habilitan el análisis del espacio más allá de las determinaciones físicas, y que amplía el concepto de lo que la Geografía denota como humano, cuestión que no solo limita el campo conceptual del futuro egresado, sino a la vez, la construcción de argumentos propios del campo disciplinar.

\section{REFERENCIAS}

Aragón, M. (2007). Las ciencias experimentales y la enseñanza bilingüe. Revista Eureka sobre Enseñanza y Divulgación de las Ciencias, 4(1), 152-175.

Archila, P. (2012) La investigación en argumentación y sus implicaciones en la formación inicial de profesores de ciencias. Revista Eureka sobre Enseñanza y Divulgación de las Ciencias, 9(3), 361-375.

Arnoux, E., Di Stefano, M. y Pereira, C. (2002). La lectura y la escritura en la universidad. Buenos. Aires: EUDEBA.

Backhoff, E., Velasco, V., y Peón, M. (2013). Evaluación de la competencia de expresión escrita argumentativa de estudiantes universitarios. Revista de la educación superior, 42(167), 9-39.

Bar, A. (2012). Argumentar y explicar en el contexto de la formación universitaria en biología. Revista Argentina de Educación Superior, 4(4), 92-113. 
Biglan, A. (1973). Relationships between subject matter characteristics and the structure and output of university departments. Journal of Applied Psychology, 57, 204-213.

Bunge, M. (1997). La causalidad: el principio de causalidad en la ciencia moderna, Buenos Aires, Argentina: Editorial Sudamericana.

Camps, A. (1995). Aprender a Escribir Textos Argumentativos: Características Dialógicas de la Argumentación Escrita. Comunicación, Lenguaje y Educación, (26), 51-63.

Camps, A. y Dolz, J. (1995). Introducción: Enseñar a Argumentar: un Desafío para la Escuela Actual. Comunicación, Lenguaje y Educación, (26), 5-8.

Canals, R. (2007). La argumentación en el aprendizaje del conocimiento social. Enseñanza de las ciencias sociales: revista de investigación, (6), 49-60.

Capdepón, F. (2004). La geografía y las distintas acepciones del espacio geográfico. Investigaciones Geográficas, (34), $141-154$.

Carlino, P. (2005). Escribir, leer y aprender en la universidad. Buenos Aires, Argentina: Fondo de Cultura Académica.

Castro, M., y Sánchez, M. (2013). La expresión de opinión en textos académicos escritos por estudiantes universitarios. Revista mexicana de investigación educativa, 18(57), 483-506.

Colle, R. (2011). El análisis de contenido de las comunicaciones. España: Sociedad Latina de Comunicación Social.

Conte, C. (2000). Algunas consideraciones sobre las unidades de Análisis de Contenido Cualitativo en Krippendorff. Argentina: Doctorado en Ciencias Sociales, FLACSO. Recuperado de https://bit.ly/2kxHW4L

Corcoran, T., Mosher, F. y Rogat, A. (2009). Learning progressions in science: An evidence-based approach to reform. New York: Center on Continuous Instructional Improvement Teachers College-Columbia University.

Cordero, M. (2000). El componente "tesis" en los textos argumentativos escolares. Revista Signos, 33(48), 87-96.

Cuadra, D. (2013). Teoría de la geografía: reflexiones en torno a la identidad de la disciplina. Perspectiva Geográfica. Revista del Programa de Estudios de Posgrado en Geografía, 18(2), 325-346.

Cuadra, D. (2014). Los enfoques de la geografía en su evolución como ciencia. Revista geográfica Digital, (21), 1-22.

Cuenca, M. (1995). Mecanismos lingüísticos y discursivos de la argumentación. Comunicación, Lenguaje y Educación, 7(2), 23-40.

Custodio, E. y Sanmartí, N. (2005). Mejorar el aprendizaje en la clase de Ciencias aprendiendo a escribir justificaciones. En VI Congreso Enseñanza de las ciencias. Conferencia llevada a cabo en Granada, España. Recuperado de ht tps://bit.ly/2kuIQih

De La Barrera, S. y Bono, A. (2004). Escribir para aprender mejor en la universidad: prácticas de escritura en contextos pedagógicos. Acción Pedagógica, 13(1), 32-36.

De Zerdán, C., De Sirgo, S. y López, E. (2010a). Competencias argumentativas en la alfabetización académica. @ tic. Revista dinnovació educativa, 4, 1-12.

De Zerdán, C., Douglas, S., y López E. (2010b). La comunicación académica como construcción argumentativa: perspectivas de lectura y de escritura de estudiantes universitarios. En V. M. Castel y L. Cubo de Severino (Eds.), La renovación de la palabra en el bicentenario de la Argentina. Los colores de la mirada lingüistica (pp. 963-970). Mendoza, Argentina: Editorial FFyL, UNCuyo.

Erduran, S. y Jiménez, M. (Ed.). (2007). Argumentation in science education: Perspectives from classroom-based research. New York: Springer.

Facultad de Humanidades-Universidad Nacional del Nordeste [UNNE] (2000). Plan de estudios de la Licenciatura en Geografia, Res $N^{\circ}$ 280/00. Resistencia, Chaco, Argentina: UNNE

Gaviria, C. y Corredor, J. (2011). Coordinación de evidencias, explicaciones y objetivos pragmáticos en la argumentación. Revista Colombiana de Psicología, 20(1), 43-56.

Gómez, J. (2006). Discurso argumentativo y auditorio. Co-herencia. Revista de Humanidades, 3(4), 9-33.

Henao, B. y Stipcich, M. (2008). Educación en ciencias y argumentación: la perspectiva de Toulmin como posible respuesta a las demandas y desafíos contemporáneos para la enseñanza de las Ciencias Experimentales. Revista Electrónica de Enseñanzas de las Ciencias, 7(1), 47-62. 
Kelly, G. y Tako, A. (2002). Epistemic levels in argument: An analysis of university oceanography students' use of evidence in writing. Science education, 86(3), 314-342.

Kuhn, D. (2010). Teaching and learning science as argument. Science Education, 94, 810-824. doi: https://doi.org/ $10.1002 /$ sce. 20395

Larraín, A., Freire, P., Moretti, R., Requena, M. y Sabat, B. (2015). La Universidad de Chile promueve las habilidades de argumentación escrita: Un estudio exploratorio comparativo de estudiantes de educación universitaria y educación técnica, Calidad en la educación, (43), 201-228.

Molina, M. y Padilla, C. (2013). Argumentar en dos disciplinas universitarias: una aproximación toulminiana a la argumentación académica en Letras y Biología. Logos: Revista de Lingüistica, Filosofía y Literatura, 23(1), 62-79.

Ordóñez, T. (1991). Cuatro enfoques en Geografía. Boletín de la Sociedad Geográfica de Colombia, 40(124), 85-106.

Padilla, C. y Carlino, P. (2010). Alfabetización académica e investigación acción: enseñar a elaborar ponencias en la clase universitaria. En Memoria Congreso Alfabetización académica y profesional en el Siglo XXI: Leer y escribir desde las disciplinas (pp. 153-182). Santiago, Chile.

Revel, A., Couló, A., Erduran, S., Furman, M., Iglesia, P. y Adúriz-Bravo, A. (Coords). (2005). Estudios sobre la enseñanza de la argumentación cientifica escolar. En VI Congreso Enseñanza de las ciencias. Conferencia llevada a cabo en Granada, España.

United Nations Educational, Scientific and Cultural Organization (UNESCO) (1995). Documento de politica para el cambio y el Desarrollo para la Educación Superior. Recuperado de http://unesdoc.unesco.org/images/0009/0 00989/098992S.pdf

United Nations Educational, Scientific and Cultural Organization (UNESCO) (1999). Declaración mundial sobre la educación superior en el siglo XXI: visión y acción y marco de acción prioritaria para el cambio y el desarrollo de la educación superior. En Conferencia Mundial Sobre La Educación. Conferencia llevada a cabo en Francia.

Van Eemeren, F., Grootendorst, R., Jackson, S., Jacobs, S. (1997). Argumentation. En T. Van Dijk, (Ed.), Discourse as Structure and Process (pp. 305- 334). London: Sage Publications, Ltda.

CC BY-NC-ND 